\title{
Application of FRS on Target Recognition
}

\author{
Donghua Gu1 ${ }^{1}$ Zhenyu Han ${ }^{1}$, Qing'e Wu ${ }^{2 *}$ \\ ${ }^{1}$ Engineering Training Center, Zhengzhou University of Light Industry, Zhengzhou, China \\ ${ }^{2}$ School of Electrical and Information Engineering, Zhengzhou University of Light Industry, Zhengzhou, China \\ Email: 435780870@qq.com, hzy196611@163.com, ${ }^{\star} w q e 969699 @ 163 . c o m$
}

How to cite this paper: Gu, D.H., Han, Z.Y. and Wu, Q.E. (2017) Application of FRS on Target Recognition. Open Journal of Applied Sciences, 7, 503-510.

https://doi.org/10.4236/ojapps.2017.710036

Received: August 25, 2017

Accepted: October 7, 2017

Published: October 10, 2017

Copyright $\odot 2017$ by authors and Scientific Research Publishing Inc. This work is licensed under the Creative Commons Attribution International License (CC BY 4.0).

http://creativecommons.org/licenses/by/4.0/

\begin{abstract}
In order to let machine better imitate thinking method of people to perform recognition and classification for fuzzy and uncertain thing, this paper puts forward a fuzzy and rough association method to deal with the problem. However, the application of fuzzy rough sets (FRS) will be introduced mainly on pattern recognition. Some related theories on FRS would be discussed, and some fuzzy rough mathematical methods on pattern-recognition will be given. Then, concrete applications of FRS on image processing and recognition will be introduced. Simulation result signifies that this fuzzy and rough association method is not only fast but also closer to nature attribute of thing for processing and recognizing image by comparing with the single neural network and other recognition device. The recognition rate is about $95.78 \%$.
\end{abstract}

\section{Keywords}

FR Proximity, Maximum Principle of Membership, Principle of Proximity, Target Recognition

\section{Introduction}

Because the features of many objective things have the uncertainties and ambiguities, the fuzzy set (FS) and the rough set (RS) had been proposed. Some relevant theories and applications of FS had been studied in [1]. Golan and Ziarko applied the RS theory to analyze the historical data of the stock for ten years [2], which studied the dependency between the stock price and the economic index. This had showed that FS and RS [3] had been applied in a very wide range fields. If the two can be united together, there will be a broader development prospects. In real life, however, concept and knowledge which people usually involved were fuzzy and ambiguous, i.e., the set $A$ in $U$ is a fuzzy set. The present question is: How to describe the set $A$ with the knowledge of the pair $(U, R)$ that is 
meaning? The fuzzy rough sets (FRS) model was introduced to answer such question.

Like single FS and RS approaches, its membership degrees are very artificial and not more accurate than FRS. When fusing old FS and new FS data of sequential process is required, single FS and RS that identify the target are not as good as target recognition of FRS with complete knowledge of FS and RS. As a flexibility of single FS and RS, single FS and RS methods are more thought to misuse than FRS method. The inferior performance of this single FS and RS relative to the FRS is shown by the simulation experiment. A main short of the single FS and RS methods stems from their lack of a systematic degree of membership update, but the FRS method has a systematic solid mechanism for degree of membership update of the weights thanks to the united union of FS and RS.

In previous works [4] [5] [6], some operations and attributes of fuzzy rough sets (FRS) were introduced. However, this paper will discuss differences of FRS, FS and RS on target recognition such that their practical difference can be identified further. Finally, simulation results are given, and the results show that FRS method is more effectual in the target recognition than single FS and RS methods. Thus, in order to carry out better task, their difference from the theory idea not only has been understood, but also it can be known what kind of application field they are fit for, respectively. The FRS relies on FR proximity principles and update of weight of degree of membership, while the update of weight had recourse to an optimal searching tree algorithm [7] [8]. The algebraic theories on FRS had been discussed in [6]. Some operations and operation laws on FRS such as union, intersection, complement, some properties of upper approximation and lower approximation had been also introduced [3] [8]. However, some applications on FRS, for example, FRS to target recognition, were not studied all along. This paper will discuss the issue in the following.

\section{FR Modeling for Target Recognition}

As an important aspect in target recognition is the image recognition, but the images obtained by the gather equipment not only include the recognized component of target, but also include other parts of non-target and some noises. Because of bright, illumination, hue and other reasons, the information of images is probably incomplete and fuzzy. These conditions will bring some difficulties to implement the feature extraction and exact matching for target image in next step, so it is necessary to perform the eliminating for some influences of side effect that are bring by the above factors. Therefore, in order to complete complex pattern recognition tasks in real-time, the FRS approach based on FRS theories proposed by the literatures [3] [6] [8] will be an effective tool in the process of fuzzy feature information processing. Thus, a mathematical modeling here will be given in the following.

\subsection{FR Recognition Model}

The definition of FR proximity will be introduced according to the next to de- 
gree definition of [3]. On the basis of this theory, this paper mainly discusses the application of FRS in engineering.

All FRS $A=\left\langle A_{L}, A_{U}\right\rangle$ on the universal set $\Gamma$ is denoted as $F R(\Gamma)$.

According to the proximity in [1], a FR proximity and a specific FR proximity are given below.

Definition 1. Assume $\{\Gamma, R\}$ to be a given approximate universal set. Let $A$, $B, C \in F R(\Gamma)$ and $\lambda=[0,1] . P(A, B)$ is called a FR proximity of FRS $A$ and $B$ if the mapping $N: F R(\Gamma) \times F R(\Gamma) \rightarrow \lambda$ satisfies the following several things:

1) $P(A, B)=P(B, A) \Leftrightarrow P\left(A_{L}, B_{L}\right)=P\left(B_{L}, A_{L}\right)$ and $P\left(A_{U}, B_{U}\right)=P\left(B_{U}, A_{U}\right)$;

2) $0 \leq P(A, B) \leq 1 \Leftrightarrow 0 \leq P\left(A_{L}, B_{L}\right) \leq 1$ and $0 \leq P\left(A_{U}, B_{U}\right) \leq 1$,

$P(\Gamma, \phi)=0 \Leftrightarrow P\left(\Gamma_{L}, \phi_{L}\right)=0$ and $P\left(\Gamma_{U}, \phi_{U}\right)=0$, where $\Gamma$ is a universal set, $\phi$ is an empty set;

3) If $A \subseteq B \subseteq C$, then

$P(A, C) \leq P(A, B) \wedge P(B, C) \Leftrightarrow P\left(A_{L}, C_{L}\right) \leq P\left(A_{L}, B_{L}\right) \wedge P\left(B_{L}, C_{L}\right)$ and $P\left(A_{U}, C_{U}\right) \leq P\left(A_{U}, B_{U}\right) \wedge P\left(B_{U}, C_{U}\right)$.

Where the $P$ is called a FR proximity function on $F R(\Gamma)$. The approximate universal set $\{\Gamma, R\}$ is called a FR proximity space.

According to the above definition, here will give a type of FR proximity as follows:

Theorem 1. If $\Gamma=\left\{u_{1}, u_{2}, \cdots, u_{n}\right\}$, then

$$
P(A, B) \stackrel{\Delta}{=} 1-\frac{1}{n} \sum_{i=1}^{n}\left|A\left(u_{i}\right)-B\left(u_{i}\right)\right|
$$

is a FR proximity of FRS $A$ and $B$, where $A=\left\langle A_{L}, A_{U}\right\rangle$,

$B=\left\langle B_{L}, B_{U}\right\rangle \in F R(\Gamma), u_{i} \in \Gamma, A\left(u_{i}\right)=\left\langle A_{L}\left(u_{i}\right), A_{U}\left(u_{i}\right)\right\rangle$, $B\left(u_{i}\right)=\left\langle B_{L}\left(u_{i}\right), B_{U}\left(u_{i}\right)\right\rangle$.

Define

$$
\begin{aligned}
A\left(u_{i}\right)-B\left(u_{i}\right) & =\left\langle A_{L}\left(u_{i}\right), A_{U}\left(u_{i}\right)\right\rangle-\left\langle B_{L}\left(u_{i}\right), B_{U}\left(u_{i}\right)\right\rangle \\
& =\left\langle A_{L}\left(u_{i}\right)-B_{L}\left(u_{i}\right), A_{U}\left(u_{i}\right)-B_{U}\left(u_{i}\right)\right\rangle
\end{aligned}
$$

In the real number region, when $U$ is a closed domain $\Gamma$, i.e., $U=\Gamma$, then

$$
P(A, B) \stackrel{\Delta}{=} 1-\frac{1}{|\Gamma|} \int_{\Gamma}|A(u)-B(u)| d u
$$

is a FR proximity of FRS $A$ and $B$, where $|\Gamma|$ is a measurement of $\Gamma$, which is a length, area or volume.

Proof: the Equality (1) is proved as follows:

1) $P\left(A_{L}, B_{L}\right)=1-\frac{1}{n} \sum_{i=1}^{n}\left|A_{L}\left(u_{i}\right)-B_{L}\left(u_{i}\right)\right|=1-\frac{1}{n} \sum_{i=1}^{n}\left|B_{L}\left(u_{i}\right)-A_{L}\left(u_{i}\right)\right|=P\left(B_{L}, A_{L}\right)$

and $P\left(A_{U}, B_{U}\right)=P\left(B_{U}, A_{U}\right)$, so $P(A, B)=P(B, A)$.

2) Since $0 \leq A_{L}\left(u_{i}\right), A_{U}\left(u_{i}\right), B_{L}\left(u_{i}\right), \quad B_{U}\left(u_{i}\right) \leq 1$, there are

$0 \leq P\left(A_{L}, B_{L}\right) \leq 1$ and $0 \leq P\left(A_{U}, B_{U}\right) \leq 1$, so $0 \leq P(A, B) \leq 1$.

Especially, $P\left(A_{L}, A_{L}\right)=1-\frac{1}{n} \sum_{i=1}^{n}\left|A_{L}\left(u_{i}\right)-A_{L}\left(u_{i}\right)\right|=1, P\left(A_{U}, A_{U}\right)=1$, so 
$P(A, A)=1$.

The same, $P\left(U_{L}, \phi_{L}\right)=1-\frac{1}{n} \sum_{i=1}^{n}\left|U_{L}\left(u_{i}\right)-\phi_{L}\left(u_{i}\right)\right|=1-1=0, \quad P\left(U_{U}, \phi_{U}\right)=0$, so $P(U, \phi)=0$.

3) If $A \subseteq B \subseteq C$, then

$\left|A\left(u_{i}\right)-C\left(u_{i}\right)\right| \geq\left|A\left(u_{i}\right)-B\left(u_{i}\right)\right|$ and $\left|A\left(u_{i}\right)-C\left(u_{i}\right)\right| \geq\left|B\left(u_{i}\right)-C\left(u_{i}\right)\right|$, so $1-\frac{1}{n} \sum_{i=1}^{n}\left|A\left(u_{i}\right)-C\left(u_{i}\right)\right| \leq 1-\frac{1}{n} \sum_{i=1}^{n}\left|A\left(u_{i}\right)-B\left(u_{i}\right)\right|$ and

$1-\frac{1}{n} \sum_{i=1}^{n}\left|A\left(u_{i}\right)-C\left(u_{i}\right)\right| \leq 1-\frac{1}{n} \sum_{i=1}^{n}\left|B\left(u_{i}\right)-C\left(u_{i}\right)\right|$

Therefore, there is $P(A, C) \leq P(A, B) \wedge P(B, C)$.

So, the Equality (1) is a FR proximity.

Similarly, the Equality (2) can be proved. At the same time, the proximity that is defined by the theorem 1 is called a FR 1-proximity.Q.E.D.

\subsection{FR Recognition Principle}

Here, two recognition methods of FRS are given. An immediate method is a max-principle of membership that applies mainly recognition of individuality. A mediate method is based on a principle of proximity that applies recognition of group model generally.

1) Maximum principle of membership

Definition 2. Assume $A^{i} \in F R(\Gamma),(i=1,2, \cdots, n)$. For $u_{0} \in \Gamma$, if there are an $i_{0}$ and an $j_{0}$ in order to make $A_{L}^{i_{0}}\left(u_{0}\right)=\max \left\{A_{L}^{1}\left(u_{0}\right), A_{L}^{2}\left(u_{0}\right), \cdots, A_{L}^{n}\left(u_{0}\right)\right\}$, and $A_{U}^{j_{0}}\left(u_{0}\right)=\max \left\{A_{U}^{1}\left(u_{0}\right), A_{U}^{2}\left(u_{0}\right), \cdots, A_{U}^{n}\left(u_{0}\right)\right\}$, then $u_{0}$ is believed to subordinate $A_{L}^{i_{0}}$ and $A_{U}^{j_{0}}$ relatively.

Moreover, according to the test need and the trial and error method, $A^{i_{0}}$ and $A^{j_{0}}$ can be determined.

\section{2) Principle of proximity}

Definition 3. Let $A^{i}, B \in F R(\Gamma),(i=1,2, \cdots, n)$. If there is an $i_{0}$ in order to let $P\left(A_{L}^{i_{0}}, B_{L}\right)=\max \left\{P\left(A_{L}^{1}, B_{L}\right), P\left(A_{L}^{2}, B_{L}\right), \cdots, P\left(A_{L}^{n}, B_{L}\right)\right\}$ be true, then $B_{L}$ is believed to most near $A_{L}^{i_{0}}$, i.e., $B_{L}$ and $A_{L}^{i_{0}}$ are believed to be congeneric. Similarly, if there is an $j_{0}$ in order to let $P\left(A_{U}^{j_{0}}, B_{U}\right)=\max \left\{P\left(A_{U}^{1}, B_{U}\right), P\left(A_{U}^{2}, B_{U}\right), \cdots, P\left(A_{U}^{n}, B_{U}\right)\right\}$ be true, then $B_{U}$ is thought to be the most close to $A_{U}^{j_{0}}$, i.e., $B_{U}$ and $A_{U}^{j_{0}}$ are believed to be congeneric. The principle is called a principle of proximity.

The same, according to the test need, $A^{i_{0}}$ and $A^{i_{0}}$ can be also determined.

\section{Application of FRS on Target Recognition}

\subsection{Recognition Method}

According to FRS theories proposed by the literatures [3] [6] [8] and considering speed influence for image processing, the maximum size of image matrix is chosen as $36,000 \times 29,800$ by experiments. The ratio of pixels no-colored to pixels colored on the grid the data processed is 7:2. For easy discussion, here we 
choose a small size with $360 \times 300$ and use a grid-matrix method by FRS function, as shown in Figure 1(a) and Figure 1(b).

First, let an image insert in a frame of box. At the same time, the frame is divided into many small grids. According to the degree of clarity of the image point in each small grid, an appropriate degree of membership $\mu_{i j}$ of the image point is given. Moreover, according to the size of the positive region that the image point appears in the small grid, the approximate accuracy of the image point is computed by $\alpha_{i j}=\frac{\left|\left(\Delta_{i j}\right)_{L}\right|}{\left|\left(\Delta_{i j}\right)_{U}\right|}$. Thus, the important parameter pair $\left(\mu_{i j}, \alpha_{i j}\right)$ is obtained. Where, $i$ and $j$ are the number of rows and columns of the grid, respectively; $\Delta_{i j}$ is the image point within the grid which is located in the $i^{\text {th }}$ row and the $j^{\text {th }}$ column; $\left(\Delta_{i j}\right)_{L}$ and $\left(\Delta_{i j}\right)_{U}$ is the lower approximate and upper approximate, respectively; $\left|\Delta_{i j}\right|$ denotes the size of the measure of $\Delta_{i j}$, that is, the area size of the small box. In this way, a fuzzy rough relation matrix $\left(\left(\mu_{i j}, \alpha_{i j}\right)\right)_{n \times m}$ can be created. Define $\mu_{i j}=1$ to denote that the image point appears clearly in the grid, and let it fill in the black. Similarly, define $\mu_{i j}=0$ to denote that the image point does not appear in the grid, and let it be white; define $0<\mu_{i j}<1$ to denote that this image point appear intangibly in the grid, and let this grid be a shadow.

By Figure 1(a) and Figure 1(b), the image (a) can be divided into $6 \times 5$ small grids. According to the fuzzy degree $\mu_{i j}$ defined of image and the rough degree computed by $\alpha_{i j}$ of target in the grid by the above discussion, the values of cells of matrix, i.e., the corresponding fuzzy rough relation matrix can be obtained as follows:

$S=\left[\begin{array}{ccccc}(0,0) & (0,0) & (1,0.5) & (1,0.9) & (1,0.4) \\ (0,0) & (1,0.3) & (1,0.6) & (1,0.1) & (1,0.2) \\ (0,0) & (1,0.2) & (1,0.5) & (0,0) & (0,0) \\ (0,0) & (0,0) & (1,0.4) & (1,0.4) & (0,0) \\ (1,0.1) & (1,0.2) & (0,0) & (1,0.8) & (1,0.3) \\ (0,0) & (1,0.5) & (1,0.6) & (1,0.7) & (1,0.1)\end{array}\right]$

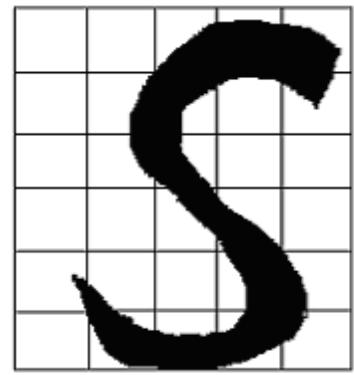

(a)

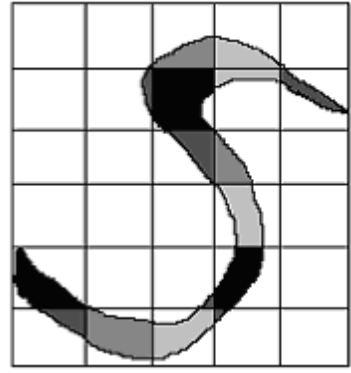

(b)

Figure 1. Known and identified image. (a) Known image; (b) Image to be identified. 
This relation matrix is called a fuzzy rough standard matrix. In order to identify the image, the outline of the image is first analyzed to see which standard image $i(i=1, \cdots, n)$ it may be belong to. The standard matrices $S_{i}(i=1, \cdots, n)$ that the $i$ images are corresponding to are usually put in sample database. Moreover, the image (b) to be identified that is shown in Figure 1 is also expressed as $6 \times 5$ fuzzy rough relation matrix $\gamma$ as an input, then the gray value information for each small grid is gotten and memorized by the photoelectric induction. By the synthesis operation of matrices and FR proximity, the FR proximity $P\left(\gamma, S_{1}\right), P\left(\gamma, S_{2}\right), \cdots, P\left(\gamma, S_{n}\right)$ between $\gamma$ and the standard matrices $S_{i}$ can be calculated. According to the selected-near principle, $\gamma$ can be determined what it is, and then the identified image can be judged to be belong to what kind of images.

\subsection{Simulation and Results Analysis}

In simulation, assume 70 known target categories have been trained, and two characteristic parameters that are $\mu_{i j}$ and $\alpha_{i j}$ have been selected. Choose randomly 100 characteristic parameters according to the uniformity distribution, and distribute equiprobably and randomly to 70 target categories. Assume the selected error of the unknown target obeys the normal distribution, and the standard variance of selected error is 3 percent of corresponding known characteristic parameter. The calculation of Formula (1) is chosen as the discriminant function for target recognition in simulation, and then after the simulation is carried out to be 160 times, the correct recognition rate that can be obtained is about $95.78 \%$ by using the FRS recognition method.

In here, in order to show the effect of FRS is better than that of single FS and RS methods on target recognition, the simulation is given. In the union of fuzzy and rough methods, the target recognition is performed. The sampling is 160 times in simulation and sampling rate $\mathrm{T}$ is 1 second. The recognition curve of FRS method comparing with FS and RS methods is shown in Figure 2.

From Figure 2, the recognition effect of FRS method is better relatively than single FS or RS methods on target recognition. The recognition curve of FRS method is basically same as the true target orbit. However, single FS and RS methods for target recognition are not so good relatively, which the difference of recognition is more. These show that the information can be almost completely applied by the FRS method than using the single FS and RS methods on the target recognition.

To error recognition curve of the difference of recognition value and true value, the recognition error of FRS method reduces gradually and trends towards stability, no matter what it is at the $x$ position direction or at the $y$ position direction. The mean-square error curve of FRS method at two-position direction is shown in Figure 3.

\section{Conclusion}

Based on the theory of FRS, this paper gives the theoretical knowledge of FR target recognition, puts forward FR proximity, and then introduces a kind of 


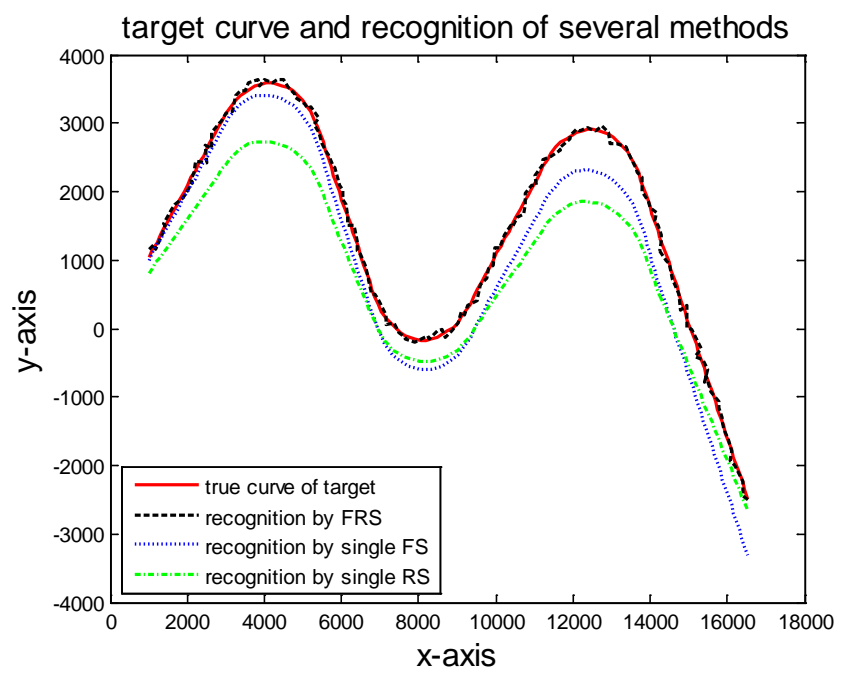

Figure 2. Comparison among FRS, single FS and single RS on target recognition.

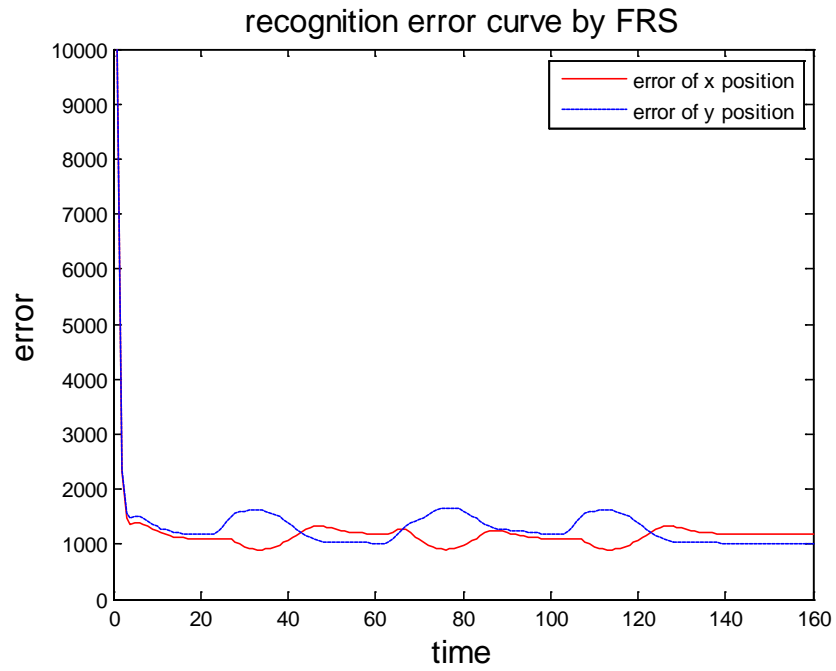

Figure 3. Recognition error by FRS method to target in both directions.

thinking method for recognition, and gives a kind of target recognition method. Finally, the application of FRS in image target recognition is discussed. To compare with FS and RS identification devices alone, the simulation results show that FRS method has faster processing speed and the processing result is closer to the natural attributes of target itself. The potentiality of FRS method in application will open up a development space based on the practical application of FRS in many areas.

\section{Acknowledgements}

This work is supported by National 973 Program (No. 613237), Henan Province Outstanding Youth on Science and Technology Innovation (No. 164100510017), respectively. 


\section{References}

[1] Yang, L.B. and Gao, Y.Y. (2002) Fuzzy Mathematics Principle and Application. University of Science and Engineering of South China Press, Guangzhou. (In Chinese)

[2] Golan, R. and Ziarko, W. (1995) Methodology for Stock Market Analysis Utilizing Rough Set Theory. Proceedings of IEEE/IAFE Conference on Computational Intelligence for Financial Engineering, New Jersey, 11-13 September 1995, 32-40. https://doi.org/10.1109/CIFER.1995.495230

[3] Nanda, S. and Majumdar, S. (1992) Fuzzy Rough Sets. Fuzzy Sets and Systems, 45, 157-160. https://doi.org/10.1016/0165-0114(92)90114-J

[4] Shamsizadeh, M. (2016) Intuitionistic General Fuzzy Automata. Soft Computing, 20, 3505-3519. https://doi.org/10.1007/s00500-015-1969-x

[5] Micic, I., Jancic, Z., Ignjatovic, J. and Ciric, M. (2015) Determinization of Fuzzy Automata by Means of the Degrees of Language Inclusion. IEEE Transactions on Fuzzy Systems, 23, 2144-2153. https://doi.org/10.1109/TFUZZ.2015.2404348

[6] Wu, Q.E., Wang, T., Huang, Y.X. and Li, J.S. (2007) New Theories on Fuzzy Rough Sets. Journal of Information and Decision Science, 5, 113-120.

[7] Garhwal, S. and Jiwari, R. (2016) Conversion of Fuzzy Automata into Fuzzy Regular Expressions Using Transitive Closure. Journal of Intelligent and Fuzzy Systems, 30, 3123-3129. https://doi.org/10.3233/IFS-152038

[8] Liu, W.N., Yao, J.T. and Yao, Y.Y. (2004) Rough Approximations under Level Fuzzy Sets. Fourth International Conference on Rough Sets and Current Trends in Computing (RSCTC2004), Uppsala, 1-5 June 2004, 78-83.

https://doi.org/10.1007/978-3-540-25929-9_8 